Article

\title{
Simultaneous Generation of Two Orthogonally Polarized Terahertz Waves by Stimulated Polariton Scattering with a Periodically Poled $\mathrm{LiNbO}_{3}$ Crystal
}

\author{
Zhongyang Li * (1), Silei Wang, Mengtao Wang, Bin Yuan and Pibin Bing ${ }^{(\mathbb{D})}$ \\ College of Electric Power, North China University of Water Resources and Electric Power, Zhengzhou 450045, \\ China; 201610521173@stu.ncwu.edu.cn (S.W.); 201610521175@stu.ncwu.edu.cn (M.W.); \\ x201710518296@stu.ncwu.edu.cn (B.Y.); bing463233@163.com (P.B.) \\ * Correspondence: thzwave@163.com; Tel.: +86-158-3827-6960
}

Received: 7 April 2018; Accepted: 20 July 2018; Published: 24 July 2018

\begin{abstract}
We present a theoretical investigation of the simultaneous generation of two orthogonally polarized terahertz $(\mathrm{THz})$ waves by stimulated polariton scattering (SPS) with a periodically poled $\mathrm{LiNbO}_{3}$ (PPLN) crystal. The two orthogonally polarized $\mathrm{THz}$ waves are generated from SPS with $A_{1}$ and $E$ symmetric transverse optical (TO) modes in a $\mathrm{LiNbO}_{3}$ crystal, respectively. The parallel polarized $\mathrm{THz}$ wave is generated from $A_{1}$ symmetric TO modes with type- 0 phase-matching of $e=e+e$, and the perpendicular polarized THz wave is generated from $E$ symmetric TO modes with type-I phase-matching of $e=o+o$. The two types of phase-matching of $e=e+e$ and $e=o+o$ can be almost satisfied simultaneously by accurately selecting the poling period of the PPLN crystal. We calculate the photon flux density of the two orthogonally polarized $\mathrm{THz}$ waves by solving the coupled wave equations. The calculation results indicate that the two orthogonally polarized $\mathrm{THz}$ waves can be efficiently generated, and the relative intensities between the two orthogonally polarized THz waves can be modulated.
\end{abstract}

Keywords: terahertz wave; stimulated polariton scattering; periodically poled $\mathrm{LiNbO}_{3}$

\section{Introduction}

Stimulated polariton scattering (SPS) has proven to be an efficient scheme to generate terahertz $(\mathrm{THz})$ waves [1-8]. A polariton is a coupled quantum between the pump laser and the infrared- and Raman-active transverse optical (TO) modes in a crystal, and it behaves like phonons near the resonant frequency associated with the TO mode and exhibits photon-like behavior for lower non-resonant frequencies [1]. SPS consists of second-order and third-order nonlinear frequency conversion processes where a pump photon stimulates a Stokes photon at the difference frequency between the pump photon and the polariton. At the same time, a THz wave is generated by the parametric process due to the nonlinearity arising from both electronic and vibrational contributions of the crystal. The TO phonon resonances can contribute substantially to the magnitude of the second-and third-order nonlinearities, which are beneficial to the THz generation via SPS.

$\mathrm{MgO}: \mathrm{LiNbO}_{3}$ has been the most widely used crystal for THz wave generation via SPS [1-5]. $\mathrm{MgO}: \mathrm{LiNbO}_{3}$ has strong second-order nonlinear response, as well as TO phonon resonances for efficient SPS [9]. MgO: $\mathrm{LiNbO}_{3}$ has five $A_{1}$ symmetric infrared- and Raman-active TO modes polarized parallel to the $c$-axis with frequencies of $248 \mathrm{~cm}^{-1}, 274 \mathrm{~cm}^{-1}, 307 \mathrm{~cm}^{-1}, 628 \mathrm{~cm}^{-1}$, and $692 \mathrm{~cm}^{-1}$ [10]. $\mathrm{MgO}: \mathrm{LiNbO}_{3}$ has eight $E$ symmetric infrared- and Raman-active TO modes polarized perpendicular to the $c$-axis with frequencies of $152 \mathrm{~cm}^{-1}, 236 \mathrm{~cm}^{-1}, 265 \mathrm{~cm}^{-1}, 322 \mathrm{~cm}^{-1}, 363 \mathrm{~cm}^{-1}, 431 \mathrm{~cm}^{-1}, 586 \mathrm{~cm}^{-1}$, and $670 \mathrm{~cm}^{-1}$ [10]. $A_{1}$ symmetric TO modes have been the most widely used for $\mathrm{THz}$ wave generation 
via SPS [1-5]. However, E symmetric TO modes can be also employed to generate THz waves via SPS. In 1969, Yarborough reported the observation of tunable SPS from $A_{1}$ and $E$ symmetric TO modes with a pump wave in a $\mathrm{LiNbO}_{3}$ crystal [11]. If the SPS from $A_{1}$ and $E$ symmetric TO modes can be simultaneously excited, then two orthogonally polarized $\mathrm{THz}$ waves can be simultaneously generated. Orthogonally polarized THz waves are useful for imaging [12]. Yu et al. [12] showed that the addition or subtraction of two images, which were taken with a perpendicularly polarized $\mathrm{THz}$ wave and parallel polarized $\mathrm{THz}$ wave, was effective to enhance the contrast of terahertz images.

In this work, we theoretically study the simultaneous generation of two orthogonally polarized $\mathrm{THz}$ waves by SPS with a periodically poled $\mathrm{LiNbO}_{3}$ (PPLN) crystal. The two orthogonally polarized $\mathrm{THz}$ waves are generated from SPS with $A_{1}$ and $E$ symmetric TO modes in a MgO:LiNbO 3 crystal, respectively. We calculate the photon flux density of the two orthogonally polarized $\mathrm{THz}$ waves by solving the coupled wave equations.

\section{Theoretical Model}

Figure 1 shows a schematic diagram of THz wave generation by the SPS processes by a PPLN crystal with a quasi-phase-matching (QPM) condition. A pump wave and two seed waves (Seed and Seed $\mathrm{o}_{\mathrm{o}}$ ) propagate along the $x$-axis of the PPLN crystal. The electric field of the pump wave and Seed $_{\mathrm{e}}$ is along the $z$-axis of the PPLN crystal, whereas the electric field of Seed $\mathrm{o}_{\mathrm{o}}$ is perpendicular to the $z$-axis of the PPLN crystal. The $z$-axis is the optical axis of the $\mathrm{LiNbO}_{3}$ crystal. The poling period of the PPLN crystal is $\Lambda$. Two orthogonally polarized $\mathrm{THz}$ waves $\left(\mathrm{THz}_{\mathrm{e}}\right.$ and $\left.\mathrm{THz}_{\mathrm{o}}\right)$ are generated by the SPS processes. The electric field of $\mathrm{THz}_{\mathrm{e}}$ is along the $z$-axis of the PPLN crystal, whereas the electric field of $\mathrm{THz}_{\mathrm{o}}$ is perpendicular to the $z$-axis of the PPLN crystal. The pump, Seed $\mathrm{e}_{\mathrm{e}}$, and $\mathrm{THz}_{\mathrm{e}}$ waves satisfy the type- 0 phase-matching of $e=e+e$, whereas the pump, Seed ${ }_{0}$, and $\mathrm{THz}_{\mathrm{O}}$ waves satisfy the type-I phase-matching of $e=o+o$. The above two types of phase-matching can also be applied to the forward SPS processes and backward SPS processes by accurately selecting the poling period $\Lambda$ of the PPLN crystal. The generated $\mathrm{THz}$ waves are deflected by parabolic mirrors, which transmit the pump and two seed waves.

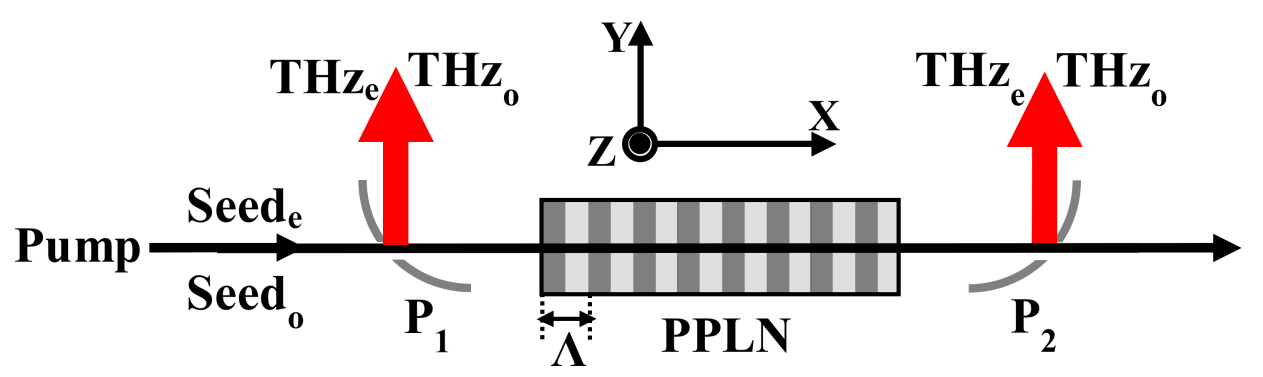

Figure 1. Schematic diagram of terahertz $(\mathrm{THz})$ wave generation by stimulated polariton scattering (SPS) processes in a periodically poled $\mathrm{LiNbO}_{3}$ (PPLN) crystal with a quasi-phase-matching (QPM) condition. $\Lambda$ is the poling period of the PPLN crystal. $\mathrm{P}_{1}$ and $\mathrm{P}_{2}$ are parabolic mirrors which transmit the pump and two seed waves, and couple out the two THz waves.

\section{Phase-Matching Characteristics}

Due to the different eigenfrequency, oscillator strength, and bandwidth of the TO modes, the two orthogonally polarized $\mathrm{THz}$ waves, $\mathrm{THz}_{\mathrm{e}}$ and $\mathrm{THz}_{\mathrm{o}}$, have different dispersion and absorption characteristics. Figure 2 shows the dispersion and absorption characteristics of the $\mathrm{THz}_{\mathrm{e}}$ and $\mathrm{THz}_{\mathrm{O}}$ waves. $n_{\mathrm{Te}}$ and $n_{\mathrm{To}}$ are the refractive indices of $\mathrm{THz}_{\mathrm{e}}$ and $\mathrm{THz} \mathrm{o}_{\mathrm{o}}$, respectively, and $\alpha_{\mathrm{Te}}$ and $\alpha_{\mathrm{To}}$ are the absorption coefficients of $\mathrm{THz}_{\mathrm{e}}$ and $\mathrm{THz}_{\mathrm{O}}$, respectively. The curves of $n_{\mathrm{Te}}$ and $\alpha_{\mathrm{Te}}$ are below the lowest $A_{1}$ symmetric TO mode, $248 \mathrm{~cm}^{-1}$, and the curves of $n_{\mathrm{To}}$ and $\alpha_{\mathrm{To}}$ are below the lowest $E$ symmetric TO mode, $152 \mathrm{~cm}^{-1}$. The theoretical parameters of the refractive index and absorption coefficient for $\mathrm{LiNbO}_{3}$ in the $\mathrm{THz}$ range are cited in [9]. From the figure, we find that $n_{\mathrm{Te}}$ and $n_{\mathrm{To}}$ are larger than 5 . 
The value of the refractive index in the $\mathrm{THz}$ range is much larger than that in the optical range, so the collinear phase-matching is impossible to realize. The absorption coefficients $\alpha_{\mathrm{Te}}$ and $\alpha_{\mathrm{To}}$ are very large, especially in the high $\mathrm{THz}$ frequency range.

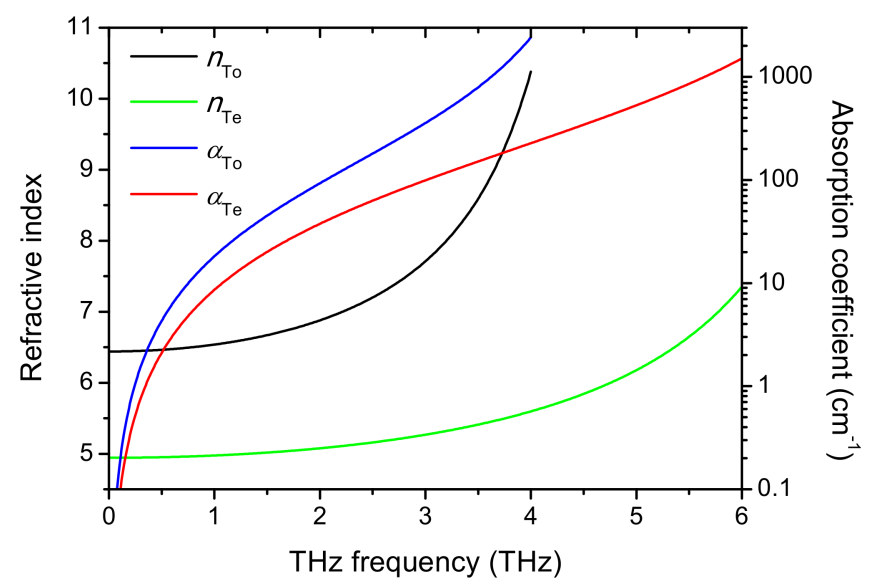

Figure 2. The dispersion and absorption characteristics of the two orthogonally polarized $\mathrm{THz}$ waves, $\mathrm{THz}_{\mathrm{e}}$ and $\mathrm{THz} z_{\mathrm{o}} \cdot n_{\mathrm{Te}}$ and $n_{\mathrm{To}}$ are the refractive indices of $\mathrm{THz}_{\mathrm{e}}$ and $\mathrm{THz} z_{\mathrm{o}}$, respectively, and $\alpha_{\mathrm{Te}}$ and $\alpha_{\mathrm{To}}$ are the absorption coefficient of $\mathrm{THz}_{\mathrm{e}}$ and $\mathrm{THz}_{\mathrm{O}}$, respectively.

In the optical SPS processes, the $\mathrm{THz}$ waves are generated, and the seed waves are amplified. The amplified seed waves are Stokes waves. In order to achieve efficient conversion of the SPS processes from the pump wave to the $\mathrm{THz}$ waves, a precise phase-matching condition must be satisfied. For the forward SPS processes, the pump, Seed $\mathrm{e}_{\mathrm{e}}$, and $\mathrm{THz}_{\mathrm{e}}$ waves satisfy the type- 0 phase-matching of $e=e+e$, and the phase mismatch $\Delta \vec{k}$ is as follows:

$$
\Delta \vec{k}_{\mathrm{e}}=\vec{k}_{p}-\vec{k}_{\mathrm{se}}-\vec{k}_{\mathrm{Te}}+\vec{k}_{\Lambda}
$$

The pump, Seed ${ }_{0}$, and $\mathrm{THz}_{\mathrm{O}}$ waves satisfy the type-I phase-matching of $e=o+o$, and the phase mismatch $\Delta \vec{k}_{\mathrm{o}}$ is as follows:

$$
\Delta \vec{k}_{\mathrm{o}}=\vec{k}_{\mathrm{p}}-\vec{k}_{\mathrm{so}}-\vec{k}_{\mathrm{To}}+\vec{k}_{\Lambda}
$$

where $\vec{k}_{\mathrm{p}}$ is the wave vector of the pump wave, $\vec{k}_{\text {se }}$ and $\vec{k}_{\text {so }}$ are the wave vectors of the two Seed and Seed ${ }_{\mathrm{o}}$ waves, respectively, and $\vec{k}_{\mathrm{Te}} \vec{k}_{\mathrm{To}}$ are the wave vectors of the two $\mathrm{THz}_{\mathrm{e}}$ and $\mathrm{THz}_{\mathrm{o}} \mathrm{waves}_{\text {, }}$ respectively. $\vec{k}_{\Lambda}=2 \pi / \Lambda$ is the grating vector, and $\Lambda$ is the poling period of the PPLN crystal.

For the backward SPS processes, the pump, Seed $\mathrm{e}_{\mathrm{e}}$ and $\mathrm{THz}_{\mathrm{e}}$ waves satisfy the type- 0 phase-matching of $e=e+e$, and the phase mismatch $\Delta \overrightarrow{k_{e}}$ is as follows:

$$
\Delta \vec{k}_{\mathrm{e}}=\vec{k}_{\mathrm{p}}-\vec{k}_{\mathrm{se}}+\vec{k}_{\mathrm{Te}}-\vec{k}_{\Lambda}
$$

The pump, seed ${ }_{\mathrm{o}}$, and $\mathrm{THz}_{\mathrm{O}}$ waves satisfy the type-I phase-matching of $e=o+o$, and the phase mismatch $\Delta \vec{k}_{\mathrm{o}}$ is as follows:

$$
\Delta \vec{k}_{\mathrm{o}}=\vec{k}_{\mathrm{p}}-\vec{k}_{\mathrm{so}}+\vec{k}_{\mathrm{To}}-\vec{k}_{\Lambda} .
$$

The energy conservation condition has to be fulfilled according to the following:

$$
\frac{1}{\lambda_{\mathrm{p}}}-\frac{1}{\lambda_{\mathrm{se}}}-\frac{1}{\lambda_{\mathrm{Te}}}=0
$$




$$
\frac{1}{\lambda_{\mathrm{p}}}-\frac{1}{\lambda_{\mathrm{so}}}-\frac{1}{\lambda_{\mathrm{To}}}=0
$$

where $\lambda_{\mathrm{p}}$ is the wavelength of pump wave, $\lambda_{\text {se }}$ and $\lambda_{\text {so }}$ are the wavelengths of the two Seed and Seed $_{\mathrm{o}}$

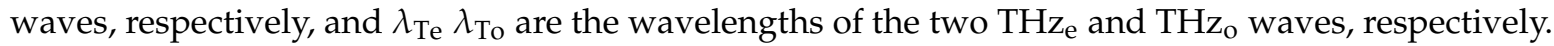
If both the phase mismatches $\Delta \vec{k}_{\mathrm{e}}$ and $\Delta \vec{k}_{\mathrm{o}}$ are small enough, two perpendicular THz waves THz and $\mathrm{THz}_{\mathrm{o}}$ can be generated simultaneously with a single pump wave.

For the SPS processes, we calculate the phase mismatches $\Delta \vec{k}$ e and $\Delta \vec{k}$ o according to Equations (1) and (2), respectively, at a fixed pump wavelength. The wavelengths of the two seed waves and the two THz waves are dependent on Equations (5) and (6). The sum phase mismatch $\Delta k_{\mathrm{s}}=\left|\Delta \overrightarrow{k_{\mathrm{e}}}\right|+\left|\Delta \vec{k}_{\mathrm{o}}\right|$. If the sum phase mismatch $\Delta k_{\mathrm{s}}$ is small enough, the two phase mismatches $\Delta \vec{k}_{\mathrm{e}}$ and $\Delta \vec{k}_{\mathrm{o}}$ are small enough to realize the two phase-matching conditions of $e=e+e$ and $e=o+o$.

Figure 3 shows the phase-matching characteristics for the forward SPS processes with a pump wavelength of $1550 \mathrm{~nm}$. $v_{\mathrm{Te}}$ and $v_{\mathrm{To}}$ are the frequencies of the $\mathrm{THz}_{\mathrm{e}}$ and $\mathrm{THz}_{\mathrm{O}} \mathrm{Waves}_{\text {, respectively. }}$ The theoretical values of the refractive indices are calculated using a Sellmeier equation for $\mathrm{LiNbO}_{3}$ in the infrared range [13]. From Figure 3a, we find that as $\Lambda$ varies from 9 to $18 \mu \mathrm{m}$, there are many points of $\Delta k_{\mathrm{s}}$ with values below $\pi \mathrm{cm}^{-1}$, which indicates that the two SPS processes generating the $\mathrm{THz}_{\mathrm{e}}$ and $\mathrm{THz}_{\mathrm{o}}$ waves can be efficiently realized. Most frequencies from 4.6 to $6 \mathrm{THz}$ of $\mathrm{THz}_{\mathrm{e}}$ and most frequencies from 0.4 to $2.8 \mathrm{THz}$ of $\mathrm{THz}_{\mathrm{o}}$ can be efficiently generated. The minimum value of $\Delta k_{\mathrm{s}}$ is $0.064 \mathrm{~cm}^{-1}$ with $\Lambda$ of $17.1 \mu \mathrm{m}$, corresponding to $\nu_{\mathrm{Te}}$ of $4.66 \mathrm{THz}$ and $\nu_{\mathrm{To}}$ of $0.56 \mathrm{THz}$. Figure $3 \mathrm{~b}$ shows the detailed phase-matching characteristics with $\Lambda$ from 17.096 to $17.100 \mu \mathrm{m}$. As $\Lambda$ varies from 17.0981 to $17.0983 \mu \mathrm{m}, \Delta k_{\mathrm{s}}$ with a value of $0.0369 \mathrm{~cm}^{-1}$ is small enough to stimulate the two SPS processes. In particular, as $\Lambda$ is $17.0982 \mu \mathrm{m}, \Delta k_{\mathrm{e}}$ equals $\Delta k_{\mathrm{o}}$, which indicates that the two SPS processes can be realized to equal degrees.

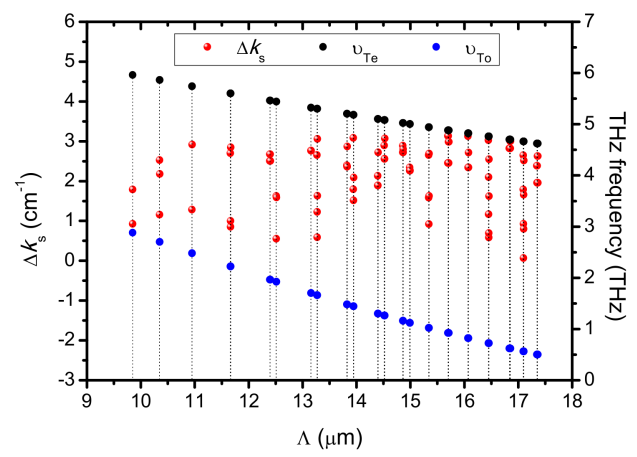

(a)

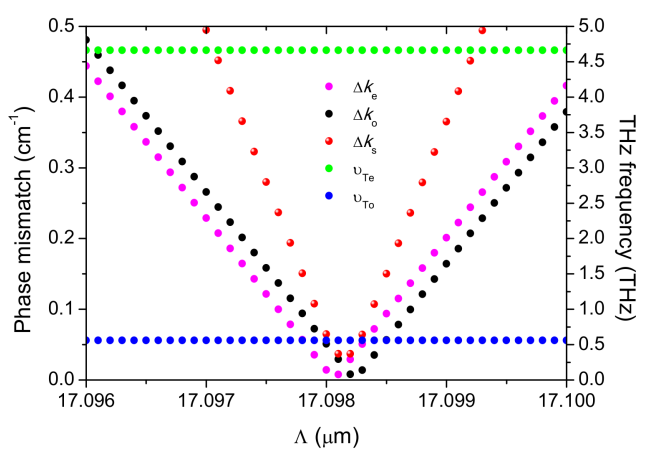

(b)

Figure 3. The phase-matching characteristics for the forward SPS processes. $v_{\mathrm{Te}}$ and $v_{\mathrm{To}}$ are the frequencies of the $\mathrm{THz}_{\mathrm{e}}$ and $\mathrm{THz} \mathrm{z}_{\mathrm{O}}$ waves, respectively. The sum phase mismatch $\Delta k_{\mathrm{s}}=\left|\Delta \vec{k}_{\mathrm{e}}\right|+\left|\Delta \vec{k}_{\mathrm{o}}\right|$, and $\lambda_{\mathrm{p}}=1550 \mathrm{~nm}$. (a) The phase-matching characteristics with $\Lambda$ from 9 to $18 \mu \mathrm{m}$. (b) The detailed phase-matching characteristics with $\Lambda$ from 17.096 to $17.100 \mu \mathrm{m}$.

Figure 4 shows the phase-matching characteristics for the backward SPS processes with a pump wavelength of $1550 \mathrm{~nm}$. From Figure $4 \mathrm{a}$, we find that as $\Lambda$ varies from 20 to $100 \mu \mathrm{m}$, there are also many points of $\Delta k_{\mathrm{s}}$ with values below $\pi \mathrm{cm}^{-1}$, particularly below $1 \mathrm{~cm}^{-1}$. Most frequencies from 0.45 to $2.03 \mathrm{THz}$ of $\mathrm{THz}_{\mathrm{e}}$ and most frequencies from 2.01 to $3 \mathrm{THz}$ of $\mathrm{THz}_{\mathrm{O}}$ can be efficiently generated. The minimum value of $\Delta k_{\mathrm{s}}$ is $0.344 \mathrm{~cm}^{-1}$ with $\Lambda$ of $80.98 \mu \mathrm{m}$, corresponding to $v_{\mathrm{Te}}$ of $0.52 \mathrm{THz}$ and $\nu_{\text {To }}$ of $2.06 \mathrm{THz}$. Figure $4 \mathrm{~b}$ shows the detailed phase-matching characteristics with $\Lambda$ around $80.98 \mu \mathrm{m}$. As $\Lambda$ varies from 80.960 to $80.995 \mu \mathrm{m}, \Delta k_{\mathrm{s}}$ with a value of $0.344 \mathrm{~cm}^{-1}$ is small enough to stimulate the 
two SPS processes. In particular, as $\Lambda$ is $80.978 \mu \mathrm{m}, \Delta k_{\mathrm{e}}$ equals $\Delta k_{\mathrm{o}}$, which indicates that the two SPS processes can be realized to equal degrees.

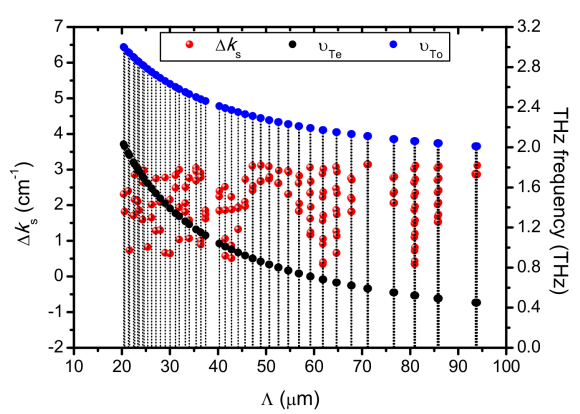

(a)

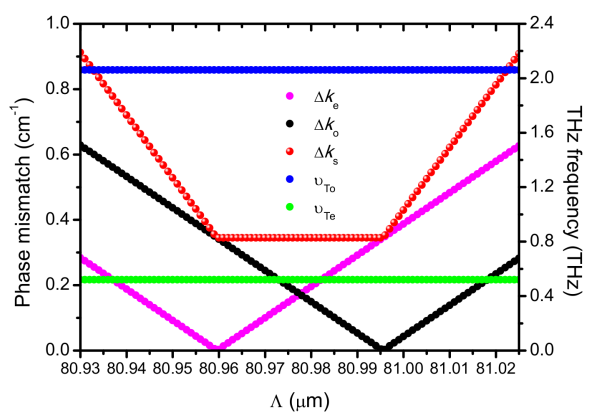

(b)

Figure 4. The phase-matching characteristics for the backward SPS processes, $\lambda_{\mathrm{p}}=1550 \mathrm{~nm}$. (a) The phase-matching characteristics with $\Lambda$ from 20 to $100 \mu \mathrm{m}$. (b) The detailed phase-matching characteristics with $\Lambda$ from 80.93 to $81.02 \mu \mathrm{m}$.

\section{THz Photon Flux Density}

The coupled wave equations for the SPS processes can be found in $[9,14]$. The coupled wave equations describe the field envelope variation of the pump, Stokes, and THz waves. The analytical expression of $\mathrm{THz}$ parametric gain coefficient $g_{\mathrm{T}}$ under the QPM condition in the international system of units can be written as follows:

$$
\begin{gathered}
g_{\mathrm{T}}=\frac{\alpha_{\mathrm{T}}}{2}\left\{\left[1+16 \cos \varphi\left(\frac{g_{0}}{\alpha_{\mathrm{T}}}\right)^{2}\right]^{\frac{1}{2}}-1\right\} \\
g_{0}^{2}=\frac{\omega_{\mathrm{s}} \omega_{\mathrm{T}}}{128 \pi^{2} \varepsilon_{0} c^{3} n_{\mathrm{p}} n_{\mathrm{s}} n_{\mathrm{T}}} I_{\mathrm{p}}\left(d_{E}+\sum_{j} \frac{S_{j} \omega_{0_{j}}^{2} d_{Q_{j}}}{\omega_{0_{j}}^{2}-\omega_{\mathrm{T}}^{2}}\right)^{2} \\
\alpha_{\mathrm{T}}=2 \frac{\omega_{\mathrm{T}}}{c} \operatorname{Im}\left(\varepsilon_{\infty}+\sum_{j} \frac{S_{j} \omega_{0_{j}}^{2}}{\omega_{0_{j}}^{2}-\omega_{\mathrm{T}}^{2}-i \omega_{\mathrm{T}} \Gamma_{j}}\right)^{\frac{1}{2}}
\end{gathered}
$$

where $\omega_{0_{j}}, S_{j}$, and $\Gamma_{j}$ denote the eigenfrequency, the oscillator strength of the polariton modes, and the bandwidth of the $j$ th TO mode in the $\mathrm{LiNbO}_{3}$ crystal, respectively. $I_{\mathrm{p}}$ is the power density of the pump wave, and $g_{0}$ is the low-loss parametric gain. $n_{\mathrm{p}}, n_{\mathrm{s}}$, and $n_{\mathrm{T}}$ are the refractive indices of the pump, Stokes, and THz waves, respectively. $\varphi$ is the angle between the wavevectors of the pump wave and THz wave. $\alpha_{\mathrm{T}}$ is material absorption coefficient in THz region. $d_{E}$ and $d_{Q}$ are nonlinear coefficients related to pure parametric (second-order) and Raman (third-order) scattering processes, respectively.

When THz frequencies are far below the lowest $A_{1}$ symmetry TO mode of $248 \mathrm{~cm}^{-1}$ and the lowest $E$ symmetry TO mode of $152 \mathrm{~cm}^{-1}$, Equation (8) can be rewritten as follows [9]:

$$
g_{0}^{2}=\frac{\omega_{\mathrm{s}} \omega_{\mathrm{T}}}{128 \pi^{2} \varepsilon_{0} c^{3} n_{\mathrm{p}} n_{\mathrm{s}} n_{\mathrm{T}}} I_{\mathrm{p}}\left(d_{E}+\sum_{j} S_{j} d_{Q_{j}}\right)^{2} .
$$

For SPS with type-0 phase-matching of $e=e+e$, the relationship between $d_{E}$ and $d_{Q}$ is given by $[9,15,16]$ the following:

$$
d_{E}+\sum_{j} S_{j} d_{Q_{j}}=\frac{1}{4} r_{33} n_{\mathrm{p}}{ }^{4}
$$


For SPS with type-I phase-matching of $e=o+o$, the relationship between $d_{E}$ and $d_{Q}$ is as follows:

$$
d_{E}+\sum_{j} S_{j} d_{Q_{j}}=\frac{1}{4} r_{13} n_{\mathbf{p}}{ }^{4}
$$

where $r_{33}$ and $r_{13}$ are the linear electro-optic coefficients of $\mathrm{LiNbO}_{3}$.

With strong $\mathrm{THz}$ wave absorption and phase mismatch and without pump depletion, the coupled wave equations can be solved to give the THz photon flux density $\phi_{\mathrm{T}}$ with a general solution [17], given by the following:

$$
\phi_{\mathrm{T}}=\phi_{\mathrm{S}}(0) e^{-\alpha_{\mathrm{T}} L / 2} \frac{g_{\mathrm{T}}^{2}}{g_{\mathrm{T}}^{2}+\left(\frac{\alpha_{\mathrm{T}}}{4}-j \frac{\Delta k}{2}\right)^{2}} \times\left|\sinh \left(\sqrt{g_{\mathrm{T}}^{2}+\left(\frac{\alpha_{\mathrm{T}}}{4}\right)^{2}} L\right)\right|^{2}
$$

where $\Delta k$ is the phase mismatching and $L$ is the crystal length. The initial $\mathrm{THz}$ photon flux density $\phi_{\mathrm{T}}$ is assumed to be zero, and $\phi_{\mathrm{S}}(0)$ is the initial seed wave photon flux density. The initial photon flux densities of Seed $\mathrm{e}_{\mathrm{e}}$ and seed ${ }_{\mathrm{o}}$ are $\phi_{\mathrm{se}}(0)$ and $\phi_{\mathrm{so}}(0)$, respectively. The THz photon flux densities of $\mathrm{THz}_{\mathrm{e}}$ and $\mathrm{THz}_{\mathrm{O}}$ are $\phi_{\mathrm{Te}}$ and $\phi_{\mathrm{To}}$, respectively. The ratio $R$ of $\phi_{\mathrm{so}}(0)$ to $\phi_{\mathrm{se}}(0)$ is as follows:

$$
R=\frac{\phi_{\mathrm{so}}(0)}{\phi_{\mathrm{se}}(0)}
$$

Figure 5 shows the THz wave photon flux densities $\phi_{\mathrm{Te}}$ and $\phi_{\mathrm{To}}$ for the forward SPS processes. From Figure $5 \mathrm{a}-\mathrm{d}$, we find that when $\Lambda$ varies from 15 to $19 \mu \mathrm{m}, \phi_{\mathrm{Te}}$ and $\phi_{\mathrm{To}}$ increase first and then decrease. When $\Lambda$ is equal to $17.0982 \mu \mathrm{m}, \phi_{\mathrm{Te}}$ and $\phi_{\mathrm{To}}$ reach their maximum values as the phase mismatches $\Delta k_{\mathrm{e}}$ and $\Delta k_{\mathrm{o}}$ reach their minimum values. The maximum value of $\phi_{\mathrm{Te}}$ is $5.74 \times 10^{-6} \mathrm{~s}^{-1} \mathrm{~cm}^{-2}$. The value of $\phi_{\mathrm{Te}}$ is so small, because the $\mathrm{THz}$ absorption coefficient of $4.66 \mathrm{THz}$ is very large. $\phi_{\mathrm{To}}$ increases with the increase of $R$. The relative photon flux densities between $\phi_{\mathrm{Te}}$ and $\phi_{\mathrm{To}}$ can be tuned by varying $R$. When $R$ is 0.0037 , the maximum values of $\phi_{\mathrm{Te}}$ and $\phi_{\mathrm{To}}$ are approximately equal. From Figure 5e, we find that when crystal length $L$ varies from 0 to $30 \mathrm{~mm}, \phi_{\mathrm{Te}}$ and $\phi_{\mathrm{To}}$ increase rapidly and smoothly. When $R$ is 0.00323 , the values of $\phi_{\mathrm{Te}}$ and $\phi_{\text {To }}$ are approximately equal, as $L$ is larger than $20 \mathrm{~mm}$.

Figure 6 shows the THz wave photon flux densities $\phi_{\mathrm{Te}}$ and $\phi_{\mathrm{To}}$ for the backward SPS processes. From Figure 6a, we find that when $\Lambda$ varies from 74 to $88 \mu \mathrm{m}, \phi_{\mathrm{Te}}$ and $\phi_{\mathrm{To}}$ increase first and then decrease. When $\Lambda$ is equal to $80.978 \mu \mathrm{m}, \phi_{\mathrm{Te}}$ and $\phi_{\text {To }}$ reach their maximum values as the phase mismatches $\Delta k_{\mathrm{e}}$ and $\Delta k_{\mathrm{o}}$ reach their minimum values. The maximum value of $\phi_{\mathrm{Te}}$ is $43.62 \mathrm{~s}^{-1} \mathrm{~cm}^{-2}$. The maximum value of $\phi_{\mathrm{Te}}$ in the backward SPS processes is larger than that in the forward SPS processes, because the $\mathrm{THz}$ absorption coefficient of $0.52 \mathrm{THz}$ in the backward SPS processes is smaller than that of $4.66 \mathrm{THz}$ in the forward SPS processes. The relative photon flux densities between $\phi_{\mathrm{Te}}$ and $\phi_{\mathrm{To}}$ can be tuned by varying $R$. When $R$ is $2.5 \times 10^{7}$, the maximum values of $\phi_{\mathrm{Te}}$ and $\phi_{\mathrm{To}}$ are approximately equal. From Figure 6b, we find that when crystal length $L$ varies from 0 to $50 \mathrm{~mm}$, $\phi_{\mathrm{Te}}$ and $\phi_{\mathrm{To}}$ increase rapidly and smoothly. When $R$ is $5.21 \times 10^{7}$ and $L$ is larger than $40 \mathrm{~mm}$, the values of $\phi_{\mathrm{Te}}$ and $\phi_{\mathrm{To}}$ are approximately equal.

The intensities of generated $\mathrm{THz}$ waves are very low, because the $\mathrm{THz}$ waves are heavily absorbed by the PPLN crystal. However, the intensities of the $\mathrm{THz}$ waves can be enhanced by injection intense seed waves, as shown in Equation (13). Moreover, one can use organic crystals with QPM, because organic crystals have larger nonlinear optical coefficients and lower absorption coefficients in the $\mathrm{THz}$ region [18]. Furthermore, the enhancement of the $\mathrm{THz}$ intensities can be realized by cryogenic cooling. At liquid nitrogen temperature, the gain coefficients of the $\mathrm{THz}$ waves in the SPS processes are enhanced. At the same time, the absorption coefficients of the $\mathrm{THz}$ waves decrease. 

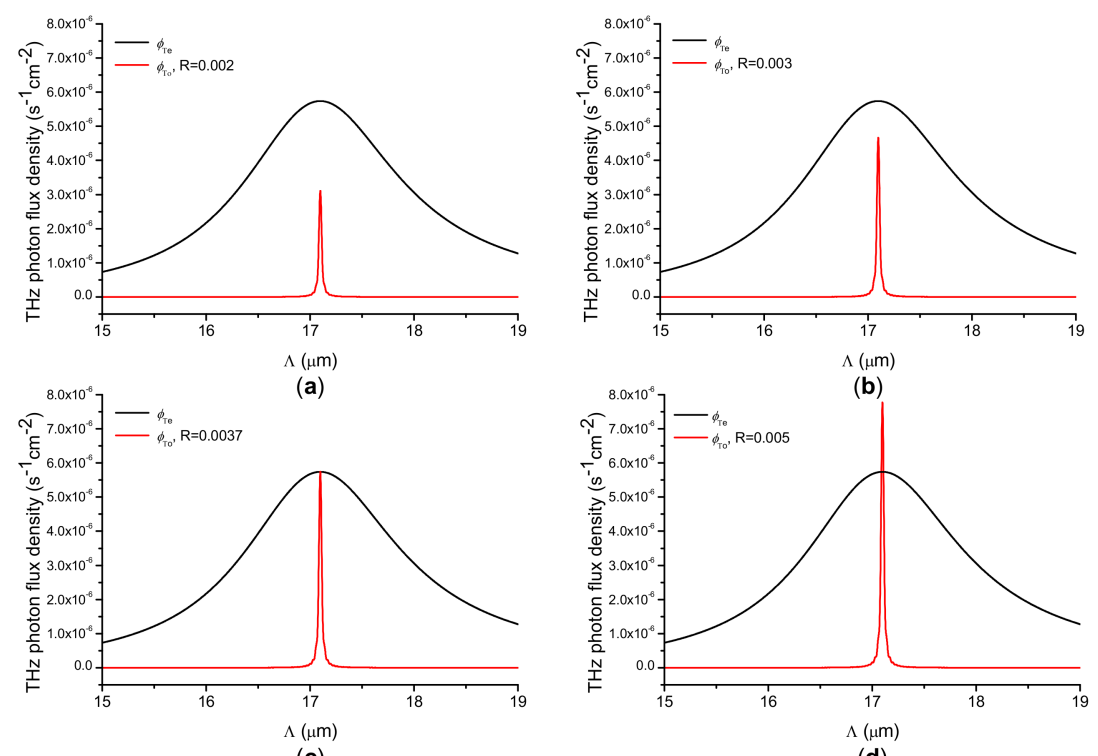

(c)

(d)

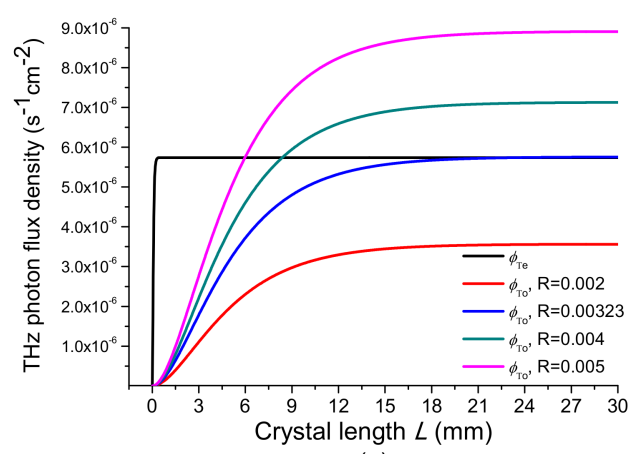

(e)

Figure 5. THz wave photon flux density $\phi_{\mathrm{T}}$ for the forward SPS processes. $\lambda_{\mathrm{p}}=1550 \mathrm{~nm}, v_{\mathrm{Te}}=4.66$ $\mathrm{THz}, v_{\mathrm{To}}=0.56 \mathrm{THz}, I_{\mathrm{p}}=100 \mathrm{MW} / \mathrm{cm}^{2}$, and $\phi_{\mathrm{se}}(0)=10^{6} \mathrm{~s}^{-1} \mathrm{~cm}^{-2}$. (a-d) $\phi_{\mathrm{T}}$ versus $\Lambda$ with $R=0.002$, $0.003,0.0037$, and 0.005 , respectively. $L=10 \mathrm{~mm}$. (e) $\phi_{\mathrm{T}}$ versus crystal length $L$. $\Lambda=17.0982 \mu \mathrm{m}$.

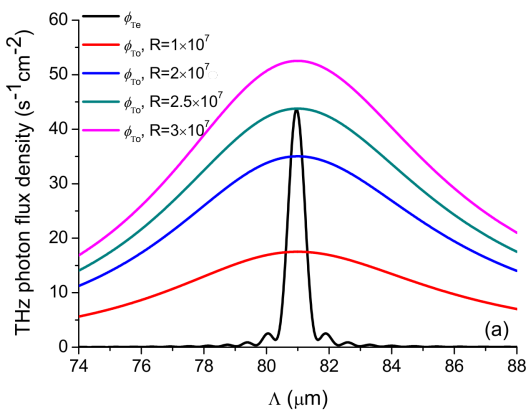

(a)

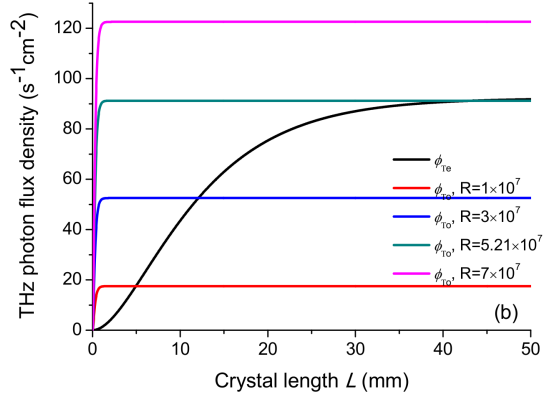

(b)

Figure 6. THz wave photon flux density $\phi_{\mathrm{T}}$ for the backward SPS processes. $\Lambda \mathrm{p}=1550 \mathrm{~nm}, v_{\mathrm{Te}}=0.52$ $\mathrm{THz}, v_{\mathrm{To}}=2.06 \mathrm{THz}, I_{\mathrm{p}}=100 \mathrm{MW} / \mathrm{cm}^{2}$, and $\phi_{\mathrm{se}}(0)=10^{6} \mathrm{~s}^{-1} \mathrm{~cm}^{-2}$. (a) $\phi_{\mathrm{T}}$ versus $\Lambda$ from 74 to $88 \mu \mathrm{m}$. $L=10 \mathrm{~mm}$. (b) $\phi_{\mathrm{T}}$ versus crystal length $L . \Lambda=80.978 \mu \mathrm{m}$.

The scheme in this work of generating two orthogonally polarized $\mathrm{THz}$ waves by SPS processes has certain advantages. First of all, the two orthogonally polarized THz waves are simultaneously 
generated by a pump wave, which means that the two $\mathrm{THz}$ waves are phase-conjugate. Second, the two orthogonally polarized $\mathrm{THz}$ waves are generated only by a PPLN crystal. Third, the intensities of the two orthogonally polarized $\mathrm{THz}$ waves can be tuned by varying the intensities of the input seed waves.

\section{Conclusions}

We present the simultaneous generation of two orthogonally polarized $\mathrm{THz}$ waves by forward and backward SPS processes with a PPLN crystal. The minimum values of $\Delta k_{\mathrm{S}}$ of $0.064 \mathrm{~cm}^{-1}$ in the forward SPS processes and $0.344 \mathrm{~cm}^{-1}$ in the backward SPS processes indicate that the type- 0 phase-matching generating parallel polarized $\mathrm{THz}$ wave and the type-I phase-matching generating perpendicular polarized THz wave can almost be satisfied simultaneously. In particular, the two SPS processes can be excited to equal degrees by accurately selecting the poling period of the PPLN crystal. We calculate the photon flux densities of the two orthogonally polarized $\mathrm{THz}$ waves by solving the coupled wave equations. The theoretical calculations show that the photon flux densities of the two orthogonally polarized $\mathrm{THz}$ waves are very small. The relative intensities between the two orthogonally polarized $\mathrm{THz}$ waves can be modulated by varying the intensities of the input seed waves.

Author Contributions: Z.L., S.W., and M.W. conceived of the original idea; B.Y. and P.B. contributed useful and deep discussions; and Z.L. wrote the manuscript. All authors read and approved the final version of the manuscript.

Funding: This work was supported by the National Natural Science Foundation of China (61601183); the Natural Science Foundation of Henan Province (162300410190); the Program for Innovative Talents (in Science and Technology) in University of Henan Province (18HASTIT023); the Young Backbone Teachers in University of Henan Province (2014GGJS-065); and the Program for Innovative Research Team (in Science and Technology) in University of Henan Province (16IRTSTHN017).

Conflicts of Interest: All contributing authors declare no conflicts of interest. The founding sponsors had no role in the design of the study; in the collection, analyses, or interpretation of the data; in the writing of the manuscript; or in the decision to publish the results.

\section{References}

1. Kawase, K.; Shikata, J.; Ito, H. Terahertz wave parametric source. J. Phys. D Appl. Phys. 2002, 35, R1-R14. [CrossRef]

2. Ikari, T.; Guo, R.; Minamide, H.; Ito, H. Energy scalable terahertz-wave parametric oscillator using surface-emitted configuration. J. Eur. Opt. Soc.-Rapid Publ. 2010, 5, 10054.

3. Ortega, T.A.; Pask, H.M.; Spence, D.J.; Lee, A.J. THz polariton laser using an intracavity Mg:LiNbO 3 crystal with protective Teflon coating. Opt. Express 2017, 25, 3991-3999. [CrossRef] [PubMed]

4. Zhang, R.; Qu, Y.; Zhao, W.; Chen, Z. High energy, widely tunable Si-prism-array coupled terahertz-wave parametric oscillator with a deformed pump and optimal crystal location for angle tuning. Appl. Opt. 2017, 56, 2412-2417. [CrossRef] [PubMed]

5. Molter, D.; Theuer, M.; Beigang, R. Nanosecond terahertz optical parametric oscillator with a novel quasi-phase-matching scheme in lithium niobate. Opt. Express 2009, 17, 6623-6628. [CrossRef] [PubMed]

6. Jang, H.; Strömqvist, G.; Pasiskevicius, V.; Canalias, C. Control of forward stimulated polariton scattering in periodically-poled KTP crystals. Opt. Express 2013, 21, 27277-27283. [CrossRef] [PubMed]

7. Jang, H.; Viotti, A.; Strömqvist, G.; Zukauskas, A.; Canalias, C.; Pasiskevicius, V. Counter-propagating parametric interaction with phonon-polaritons in periodically poled $\mathrm{KTiOPO}_{4}$. Opt. Express 2017, 25, 2677-2686. [CrossRef] [PubMed]

8. Wu, M.; Chiu, Y.; Wang, T.; Zhao, G.; Zukauskas, A.; Laurell, F.; Huang, Y. Terahertz parametric generation and amplification from potassium titanyl phosphate in comparison with lithium niobate and lithium tantalate. Opt. Express 2016, 24, 25964-25973. [CrossRef] [PubMed]

9. Sussman, S.S. Tunable Light Scattering from Transverse Optical Modes in Lithium Niobate; Microwave Laboratory Report 1970, No. 1851; Stanford University: Stanford, CA, USA, 1970.

10. Barker, A.S., Jr; Ballman, A.A.; Ditzenberger, J.A. Infrared study of the lattice vibrations in $\mathrm{LiNbO}_{3}$. Phys. Rev. B 1970, 2, 4233-4239. [CrossRef] 
11. Gelbwachs, J.; Pantell, R.H.; Puthoff, H.E.; Yarborough, M.J. A tunable stimulated Raman oscillator. Appl. Phys. Lett. 1969, 14, 258-262. [CrossRef]

12. Yu, X.; Endo, M.; Ishibashi, T.; Shimizu, M.; Kusanagi, S.; Nozokido, T.; Bae, J. Orthogonally polarized terahertz wave imaging with real-time capability for food inspection. In Proceedings of the IEEE Microwave Conference (APMC), Nanjing, China, 6-9 December 2015; Volume 2, pp. 1-3.

13. Dolev, I.; Ganany-Padowicz, A.; Gayer, O.; Arie, A.; Mangin, J.; Gadret, G. Linera and nonlinear optical properities of $\mathrm{MgO}: \mathrm{LiNbO}_{3}$. Appl. Phys. B 2009, 96, 423-432. [CrossRef]

14. Walsh, D.A. Intracavity Terahertz Optical Parametric Oscillators. Doctoral Dissertation, University of St Andrews, St Andrews, Scotland, 2011.

15. Yariv, A. Quantum Electronics, 3rd ed.; Wiley: New York, NY, USA, 1988; Chapter 16.

16. Johnston, W.D.; Kaminow, I.P. Contributions to Optical Nonlinearity in GaAs as Determined from Raman Scattering Efficiencies. Phys. Rev. 1969, 188, 1209-1211. [CrossRef]

17. Kitaeva, G.K.; Penin, A.N. Parametric frequency conversion in layered nonlinear media. J. Exp. Theor. Phys. 2004, 98, 272-286. [CrossRef]

18. Majkić, A.; Zgonik, M.; Petelin, A.; Jazbinšek, M.; Ruiz, B.; Medrano, C.; Günter, P. Terahertz source at $9.4 \mathrm{THz}$ based on a dual-wavelength infrared laser and quasi-phase matching in organic crystals $\mathrm{OH} 1$. Appl. Phys. Lett. 2014, 105, 141115. [CrossRef]

(C) 2018 by the authors. Licensee MDPI, Basel, Switzerland. This article is an open access article distributed under the terms and conditions of the Creative Commons Attribution (CC BY) license (http://creativecommons.org/licenses/by/4.0/). 